\title{
The Tandem-L Mission Proposal: Monitoring Earth's Dynamics with High Resolution SAR Interferometry
}

\author{
G. Krieger ${ }^{1}$, I. Hajnsek ${ }^{1}$, K. Papathanassiou ${ }^{1}$, M. Eineder ${ }^{2}$, M. Younis ${ }^{1}$, F. De Zan ${ }^{1}$, P. Prats ${ }^{1}$, \\ S. Huber ${ }^{1}$, M. Werner ${ }^{1}$, H. Fiedler ${ }^{1}$, A. Freeman ${ }^{5}$, P. Rosen ${ }^{5}$, S. Hensley ${ }^{5}$, W. Johnson ${ }^{5}$, L. Veilleux ${ }^{5}$, \\ B. Grafmueller ${ }^{4}$, R. Werninghaus ${ }^{3}$, R. Bamler $^{2}$, A. Moreira ${ }^{1}$ \\ ${ }^{1}$ Microwaves and Radar Institute, ${ }^{2}$ Remote Sensing Technology Institute, ${ }^{3}$ German Space Agency \\ ${ }^{1,2,3}$ German Aerospace Center (DLR) \\ ${ }^{4}$ EADS Astrium GmbH, Friedrichshafen, Germany \\ ${ }^{5}$ Jet Propulsion Laboratory, Pasadena, CA, USA
}

\begin{abstract}
Tandem-L is a proposal for an innovative interferometric and polarimetric radar mission that enables the systematic monitoring of dynamic processes on the Earth surface. Important mission objectives are global forest height and biomass inventories, large scale measurements of millimetric displacements due to tectonic shifts, and systematic observations of glacier movements. The innovative mission concept and the high data acquisition capacity of Tandem- $L$ provide a unique data source to observe, analyze and quantify the dynamics of a wide range of mutually interacting processes in the bio-, litho-, hydro- and cryosphere. By this, Tandem-L will be an essential step to advance our understanding of the Earth system and its intricate dynamics.

This paper provides an overview of the Tandem-L mission concept and its main application areas. Performance predictions show the great potential of Tandem- $L$ to acquire a wide range of bio- and geophysical parameters with high accuracy on a global scale. Innovative aspects like the employment of advanced digital beamforming techniques to improve performance and coverage are discussed in detail.
\end{abstract}

\section{INTRODUCTION}

Tandem-L is a German proposal for an innovative interferometric radar mission to monitor the Earth system and its intricate dynamics. Important mission objectives are global inventories of forest height and above-ground biomass, largescale measurements of Earth surface deformations due to plate tectonics, erosion and anthropogenic activities, observations of glacier movements and 3-D structure changes in land and sea ice, and the monitoring of ocean surface currents. The Tandem$\mathrm{L}$ mission concept is based on co-flying two fully-polarimetric L-band SAR satellites in a close formation. The synergistic use of two satellites enables highly accurate interferometric measurements to derive contiguous 3-D structure profiles and their spatiotemporal evolution. The advanced imaging capabilities and the systematic data acquisition strategy make Tandem-L a unique observatory to significantly advance our scientific understanding of environmental processes in the bio-, geo-, cryo-, and hydrosphere. A detailed description of the mission goals can be found in [1]. The German Tandem-L mission proposal has in its primary science objectives several commonalities with the DESDynI mission [2] suggested by the

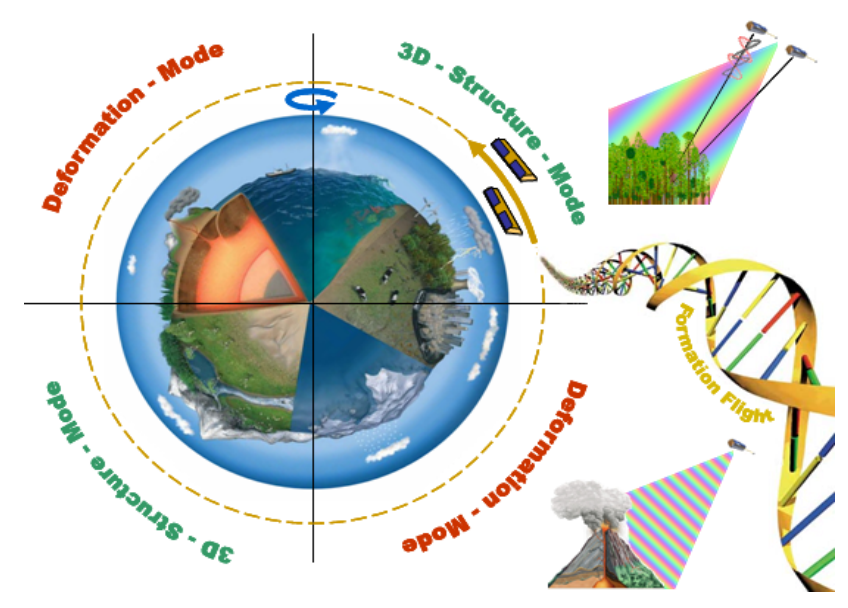

Figure 1: The Tandem-L mission concept relies on a systematic data acquisition strategy using a pair of L-band SAR satellites flying in close formation. The satellite system is operated in two basic data acquisition modes: 3-D structure mode and deformation mode. New SAR imaging techniques enable frequent coverage with high geometric resolution.

US National Research Council in its Decadal Survey for Earth Science. DLR and NASA/JPL are currently investigating in the scope of a pre-phase A study the feasibility of a joint mission that meets or even exceeds the science requirements of both proposals and provides at the same time a significant cost reduction for each partner.

Tandem-L employs many innovative techniques and technologies to achieve its ambitious mission goals. One example is the use of advanced digital beamforming techniques to provide wide swath coverage and short revisit time without sacrificing fine geometric resolution. This innovation enables a frequent monitoring of 2-D and 3-D structure changes with high precision. The systematic acquisition of wide-area single-pass and repeat-pass interferograms with high revisit frequency will open a new era in radar remote sensing and it can be expected that besides the primary mission objectives a wealth of new applications will emerge from the unique Tandem-L observatory. This paper gives an overview of the Tandem-L mission concept. Special emphasis is put on the innovative SAR imaging techniques. 


\section{SCIENCE REQUIREMENTS}

The science requirements for Tandem- $\mathrm{L}$ have been elaborated and repeatedly refined during several international workshops that brought together representatives from multiple geoscience disciplines. It turned out that Tandem-L has exceptional capabilities to acquire a worldwide unique data set that enables a wealth of innovative remote sensing applications and provides fundamental information to resolve urgent scientific questions related to climate research, geophysics, hydrology, glaciology, and vegetation monitoring. Leading scientists specified the observational requirements of their respective disciplines. The primary Tandem-L mission goals can be grouped by the following target areas and applications:

- Biosphere (3-D vegetation monitoring):

- measurement of forest height and structure

- global inventory of above ground forest biomass

- detecting vegetation disturbances and biomass changes

- Geo-/Lithosphere (deformation measurements):

- understanding earthquake and volcano eruption cycles

- quantifying the magnitude of events

- determination and forecasting the probability of events

- Hydro- \& Cryosphere (structure \& deformation):

- measurements of ice structure and its changes

- monitoring soil moisture and surface water changes

- observation of ocean currents and wave field dynamics

The reader is referred to [1] for a description of the Tandem-L science objectives. Table 1 provides a short summary of a selected subset of the collected requirements.

TABLE I. TANDEM-L USER REQUIREMENTS (EXCERPT)

\begin{tabular}{|c|c|c|c|c|}
\hline & Science Product & Coverage & $\begin{array}{l}\text { Product } \\
\text { Resolution }\end{array}$ & $\begin{array}{l}\text { Product } \\
\text { Accuracy }\end{array}$ \\
\hline \multirow{4}{*}{  } & Forest Height & \multirow{4}{*}{$\begin{array}{c}\text { all } \\
\text { forest } \\
\text { areas }\end{array}$} & $\begin{array}{l}50 \mathrm{~m} \text { (global) } \\
20 \mathrm{~m} \text { (local) }\end{array}$ & $\sim 10 \%$ \\
\hline & Above Ground Biomass & & $\begin{array}{l}100 \mathrm{~m} \text { (global) } \\
50 \mathrm{~m} \text { (regional) }\end{array}$ & $\begin{array}{c}\sim 20 \% \\
\text { (or } 20 \text { t/ha) }\end{array}$ \\
\hline & $\begin{array}{l}\text { Vertical Forest } \\
\text { Structure }\end{array}$ & & $\begin{array}{l}50 \mathrm{~m} \text { (global) } \\
20 \mathrm{~m} \text { (local) }\end{array}$ & 3 layers \\
\hline & Underlying Topography & & $50 \mathrm{~m}$ & $<4 \mathrm{~m}$ \\
\hline \multirow{4}{*}{ 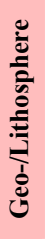 } & Plate Tectonics & $\begin{array}{l}\text { all risk } \\
\text { areas }\end{array}$ & $\begin{array}{l}100 \mathrm{~m} \text { (global) } \\
<20 \mathrm{~m} \text { (fault) }\end{array}$ & $\begin{array}{l}1 \mathrm{~mm} / \mathrm{year} \\
\text { (after } 5 \mathrm{y})\end{array}$ \\
\hline & Volcanoes & $\begin{array}{l}\text { all land } \\
\text { volcanoes }\end{array}$ & $20-50 \mathrm{~m}$ & $5 \mathrm{~mm} /$ week \\
\hline & Landslides & risk areas & $5-20 m$ & $5 \mathrm{~mm} /$ week \\
\hline & Subsidence & urban areas & $5-20 m$ & $1 \mathrm{~mm} /$ year \\
\hline \multirow{6}{*}{ 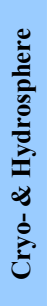 } & Glacier Flow & $\begin{array}{c}\text { main } \\
\text { glaciers }\end{array}$ & $100-500 \mathrm{~m}$ & $\begin{array}{l}5-50 \\
\text { m/year }\end{array}$ \\
\hline & Soil Moisture & selected areas & $50 \mathrm{~m}$ & $5-10 \%$ \\
\hline & Water Level Change & regional & $50 \mathrm{~m}$ & $10 \mathrm{~cm}$ \\
\hline & Snow Water Equivalent & local (exp.) & $100-500 \mathrm{~m}$ & $10-20 \%$ \\
\hline & Ice Structure Changes & local (exp.) & $100 \mathrm{~m}$ & > 1 layer \\
\hline & Ocean Currents & prio. areas & $\sim 100 \mathrm{~m}$ & $<1 \mathrm{~m} / \mathrm{s}$ \\
\hline$\sum$ & $\begin{array}{l}\text { Digital Terrain \& } \\
\text { Surface Model }\end{array}$ & Global & $\begin{array}{l}\sim 20 \mathrm{~m} \text { (bare) } \\
\sim 50 \mathrm{~m} \text { (forest) }\end{array}$ & $\begin{array}{l}2 \mathrm{~m} \text { (bare) } \\
4 \mathrm{~m} \text { (veg.) }\end{array}$ \\
\hline
\end{tabular}

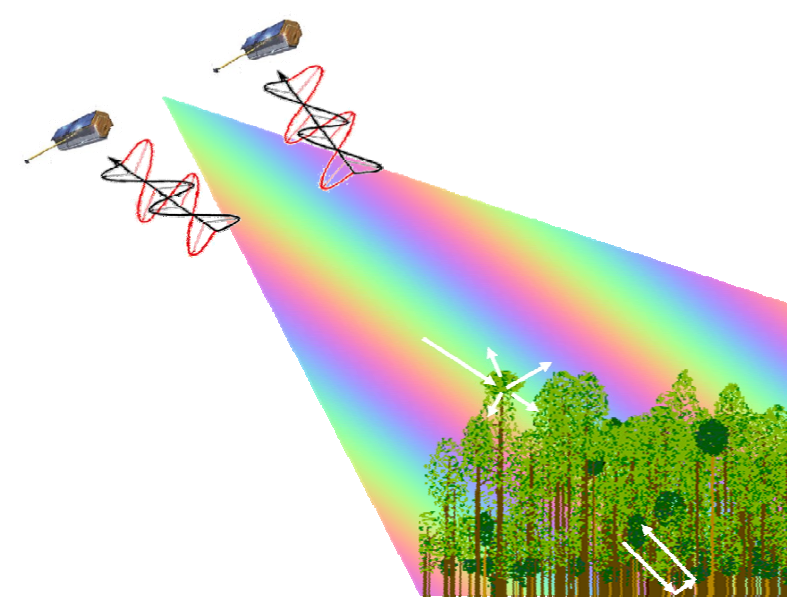

Figure 2: The 3-D structure mode employs polarimetric SAR interferometry to measure tree heights and vertical vegetation profiles. The individual contributions from ground and canopy are separated via their polarimetric signatures and the corresponding heights are measured by cross-track interferometry.

\section{MISSION CONCEPT}

The Tandem-L mission concept relies on a systematic data acquisition strategy using a pair of co-operating L-band SAR satellites flying in close formation. The satellite system operates in two basic measurement modes:

- The 3-D structure mode employs fully-polarimetric single-pass SAR interferometry to acquire structural parameters and quasi-tomographic images of semitransparent volume scatterers like vegetation, sand, and ice (cf. Figure 2).

- The deformation mode employs repeat-pass interferometry in an ultra-wide swath mode to measure small shifts on the Earth surface with millimetric accuracy and short repetition intervals.

Figure 3 shows the predicted accuracy of 1-D line-of-sight displacements in the deformation mode. Here, a linear deformation model with parameters provided in the figure caption has been assumed. It becomes clear that, for a large number of images, contributions from SNR decorrelation can be neglected while temporal decorrelation and atmospheric phase errors become the main limiting factors. Errors from temporal decorrelation can be reduced by increasing the number of independent looks, while the highly correlated atmospheric errors ask for an increased number of acquisitions ${ }^{1}$. Anyway, a large number of high resolution SAR images will be necessary to achieve the desired accuracy of $1 \mathrm{~mm} /$ year after a 5 to 7 -year mission time. 2-D deformation measurements require in addition observations from ascending and descending orbits. Tandem-L improves the deformation measurements via innovative SAR modes that enable frequent coverage with high geometric resolution (cf. Sect. IV).

\footnotetext{
${ }^{1}$ Contributions from the ionosphere can also be reduced by advanced calibration techniques while contributions from the troposphere could be reduced and/or filtered via external (e.g. meteorological) information.
} 


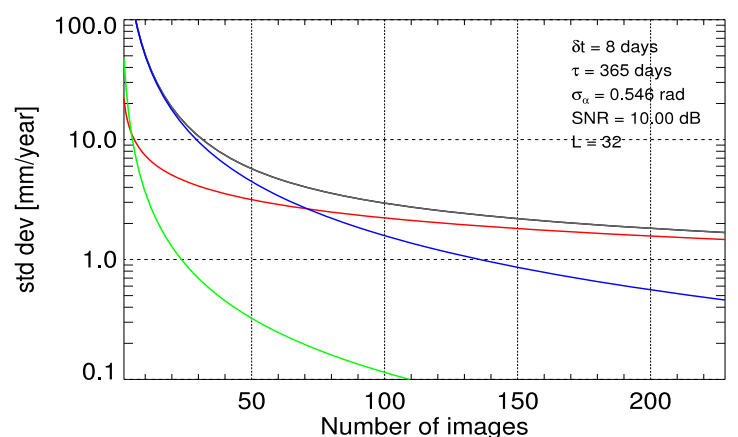

Figure 3: Performance prediction example for the deformation mode. The coloured curves show the individual error contributions from SNR decorrelation (green), atmosphere (blue), and temporal decorrelation (red). The overall error is shown in grey. The model in [6] has been used assuming a repeat orbit of $\delta \mathbf{t}=\mathbf{8}$ days, an exponential coherence decay with $\tau=\mathbf{3 6 5}$ days, an atmospheric phase error standard deviation of 0.546 $\operatorname{rad}(\sim 10 \mathrm{~mm} @ \mathrm{~L}-\mathrm{band})$, an SNR of $10 \mathrm{~dB}$, and $\mathrm{L}=32$ independent looks.

Figure 4 shows an example of the predicted accuracy of forest height measurements using the Pol-InSAR structure mode [4][5]. The performance depends on both the forest height and the length of the cross-track baseline (expressed here in terms of the vertical wavenumber $k_{z}$ ). Accuracies below $10 \%$ can be achieved by combining multiple acquisitions with different vertical wavenumbers.

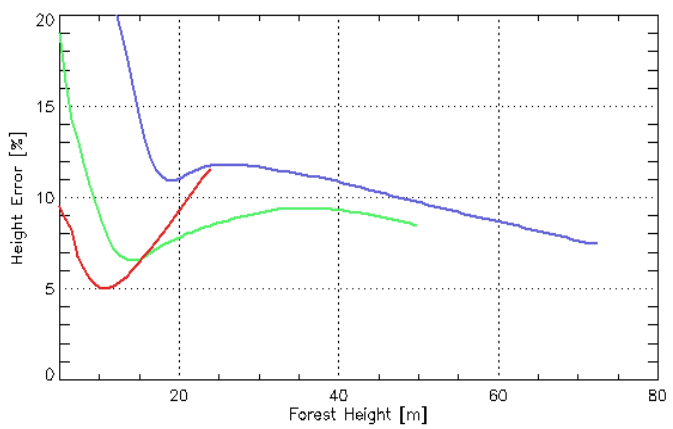

Figure 4: Performance prediction example for the 3-D structure mode. The coloured curves show the expected accuracy of vegetation height measurements for different vertical wavenumbers (blue: $\boldsymbol{k}_{z}=0.05 \mathrm{rad} / \mathrm{m}$ green: $k_{z}=0.1 \mathrm{rad} / \mathrm{m}$, red: $k_{z}=0.2 \mathrm{rad} / \mathrm{m}$ ) and $\mathrm{n}=30$ looks. A random volume over ground model has been assumed with an extinction of 0.3 $\mathrm{dB} / \mathrm{m}$. System errors (mainly from limited SNR) have been modeled by a decorrelation factor of $\gamma_{s y s}=\mathbf{0 . 9}$. The domain of the individual curves is limited to those forest heights that can be measured without ambiguities.

The Tandem-L acquisition plan foresees a systematic variation of the cross-track baselines to optimize forest height and vegetation profile measurements in the 3-D structure mode. At least three acquisitions with vertical wavenumbers $k_{z}$ ranging from $0.05 \mathrm{rad} / \mathrm{m}$ to $0.2 \mathrm{rad} / \mathrm{m}$ are planned for each season. Figure 5 shows the correspondence between $k_{z}$ and the lengths of the perpendicular baselines assuming an interferometric acquisition in bistatic mode. For an orbital altitude of $700 \mathrm{~km}$ and incident angles ranging from $30^{\circ}$ to $45^{\circ}$, the required perpendicular baselines vary between $750 \mathrm{~m}$ and $5 \mathrm{~km}$. At the equator, this corresponds to horizontal baselines between $850 \mathrm{~m}$ and $6.6 \mathrm{~km}$ in case of using a Helix formation [3] with no radial orbit separation at zero latitude.

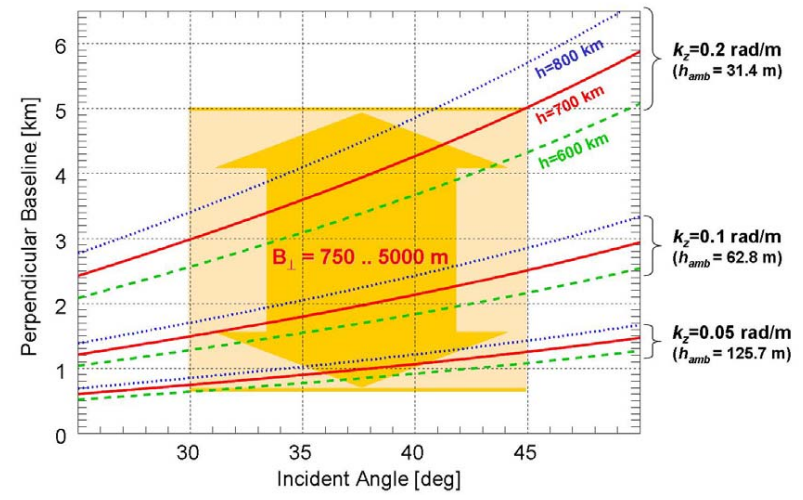

Figure 5: Correspondence between vertical wavenumbers and perpendicular baselines as a function of the incident angle and the orbital altitude.

An elegant technique to provide this wide range of crosstrack baselines exploits the naturally occurring differential secular variations of the right ascension of the ascending nodes in response to slightly different inclinations. Figure 6 shows the evolution of the horizontal baselines at the equator for different inclination offsets (expressed as horizontal baselines at the northern and southern orbit turns).
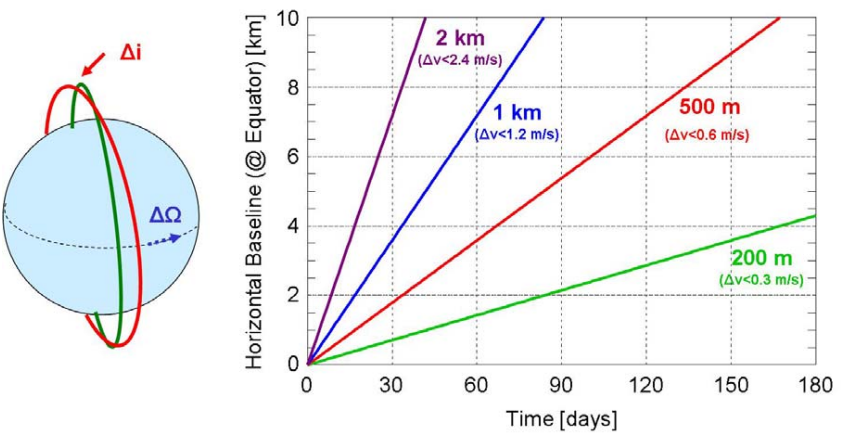

Figure 6: A systematic variation of the equatorial cross-track baselines can be achieved by using orbits with slightly different inclinations. The inclination offset causes a relative drift of the ascending nodes.

A further challenge for Tandem- $\mathrm{L}$ is the adjustment of large cross-track baselines at higher latitudes. One opportunity is the use of a large eccentricity offset to provide a sufficient radial orbit separation at high latitudes, but a significant amount of fuel ${ }^{2}$ will be required to compensate the resulting motion of libration for longer time periods. Another opportunity is the use of an even larger separation of the ascending nodes, which may then provide sufficient baselines for accurate forest height retrievals in the mid latitudes. Boreal forests at higher latitudes can moreover be imaged in the alternating bistatic mode [3] which doubles the phase to height scaling, thereby increasing the effective baseline by a factor of two. An optimized data acquisition concept is currently under development.

\footnotetext{
${ }^{2}$ The required $\Delta v$ is about $10 \mathrm{~m} / \mathrm{s}$ per $\mathrm{km}$ radial baseline for each year. A 5 -year mission with an average radial baseline of $2 \mathrm{~km}$ would hence require about $5 \%$ of the satellite mass for fuel in case of using a hot gas propulsion system with an exhaustive velocity of $2000 \mathrm{~m} / \mathrm{s}$ and more than $20 \%$ would be required in case of using a cold gas system with $500 \mathrm{~m} / \mathrm{s}$ exhaust velocity.
} 


\section{SAR INSTRUMENT INNOVATION}

A challenge for Tandem- $\mathrm{L}$ is the development of costeffective and - at the same time - powerful SAR satellites that meet the high demands from the science requirements. The achievement of this goal requires new ideas, concepts and technologies. A key innovation in Tandem- $\mathrm{L}$ will be a shift of the digital interface towards the radar antenna. The software controlled generation of multiple and highly adaptive antenna beams allows for novel and extremely powerful SAR imaging modes that can be optimally adapted to the different user requirements.

The current Tandem-L design foresees an 8-day repeat orbit with an altitude of $760 \mathrm{~km}$, but there is still the option to lengthen the repeat cycle in favor of the ability to cover each area with at least two different incident angles. In any case, a minimum swath width of $350 \mathrm{~km}$ will be required to provide full spatial coverage at the equator without loosing satellite passes for high precision repeat-pass interferometry.

The imaging of wide swaths with reasonable range and azimuth resolutions requires an extremely capable SAR instrument with a large antenna aperture. Two basic designs are currently being investigated for Tandem-L. The first design is based on a planar direct radiating array, while the second design employs a deployable reflector antenna. A deployable reflector has the great advantage to provide a large aperture at low weight and cost, but it may also require a more demanding orbit and attitude steering.

\section{A. Digital Beamforming with Reflector Antennas}

Unfurlable reflectors are now a mature technology with extensive flight heritage in space telecommunications and lightweight mesh reflectors with diameters of $20 \mathrm{~m}$ and more will be deployed in space in the near future. The mesh surface accuracies can meet requirements beyond Ka-band and are hence more than sufficient for an L-band radar mission. A very powerful SAR instrument architecture arises if a large paraboloidal reflector is combined with a digital feed array as illustrated schematically in Figure 7 (cf. [7], [8]).

The simultaneous activation of all feed elements generates a broad transmit beam without spill-over as desired for wide swath illumination. On the other hand, radar echoes arriving as plane waves from a given direction activate typically only one or a small number of feed elements if the feed array is located close to the focal plane. This systematic correspondence between beam direction and activated feed array element(s) is well suited to significantly enhance the imaging performance of Tandem- $\mathrm{L}$ without an undue increase of the implementation complexity and the costs of the radar instrument. For example, a significant improvement in NESZ and range ambiguity suppression is achieved via a dynamic routing of the individual feed signals. The selective routing corresponds to a real-time digital beamforming where a narrow antenna beam with high receiver gain is steered in synchrony with the systematic variation of the swath echoes' direction of arrival. The real-time beamsteering makes full use of the available antenna aperture for high-gain signal reception

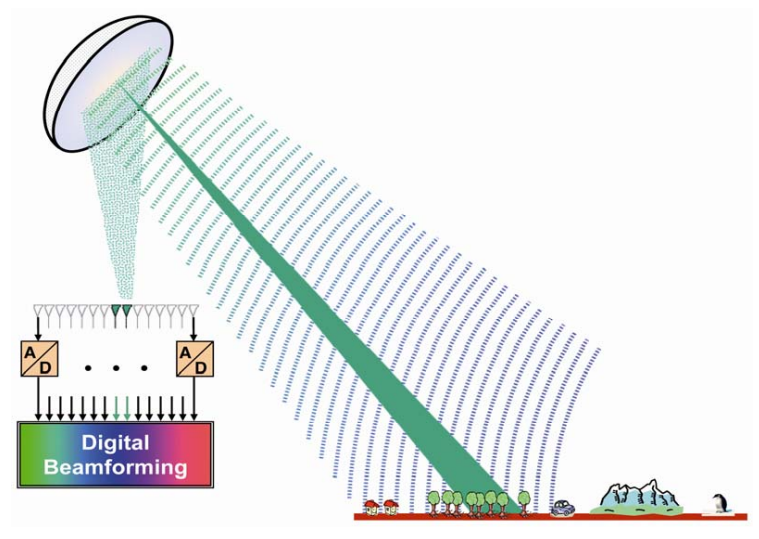

Figure 7: Digital beamforming with large reflector antennas.

without loosing the opportunity for wide swath coverage. The increased performance relaxes the thermal, power and energy demands and/or allows for longer operation times as desired for a systematic Earth monitoring mission.

\section{B. Wide-Swath Imaging with High Azimuth Resolution}

The digital beamforming architecture of Tandem-L can moreover improve the spatial coverage without reducing the geometric resolution. High resolution acquisitions are, e.g., desired for systematic deformation measurements in urban areas, land slides and the immediate neighborhood of faults, for ice flow velocity measurements and for 3-D structure measurements via polarimetric SAR interferometry, where a higher geometric resolution allows for an increased number of looks to improve the retrieval of vegetation parameters. The classical solution to wide-swath SAR imaging is ScanSAR, where the coverage is improved at the cost of an impaired azimuth resolution. Digital beamforming allows for new acquisition modes that avoid the resolution compromise of the ScanSAR technique. One example is the simultaneous imaging of multiple swaths [7]. For a reflector antenna, multiple beams can easily be formed by recording simultaneously displaced subsets of activated feed element signals. The simultaneous recording of multiple swaths allows for an improved coverage without deteriorating the azimuth resolution (as in the ScanSAR mode). The different swaths are separated by "blind ranges", as the radar cannot receive radar echoes while transmitting. Due to the high receiver gain, these gaps can be made rather small. Figure 8 shows a performance example for the imaging of a $350 \mathrm{~km}$ wide swath in full polarization using a four-beam stripmap mode.

TABLE II. SUMMARY OF SYSTEM PARAMETERS USED IN FIGURE 8

\begin{tabular}{lclc}
\hline Parameter & Value & Parameter & Value \\
\hline Orbit Height & $760 \mathrm{~km}$ & Reflector Size & $15 \mathrm{~m}$ (diameter) \\
Repeat Cycle & 8 days & Focal Length & $10 \mathrm{~m}$ \\
Incident Angle & $26.3^{\circ}-46.6^{\circ}$ & Feed Location & centre \\
Look Angle & $23.3^{\circ}-40.5^{\circ}$ & Feed Elements & 24 \\
Swath Width & $349 \mathrm{~km}$ & Feed Length & $3.43 \mathrm{~m}$ \\
Ground Range & $331-680 \mathrm{~km}$ & Tilt Angle & $31.9^{\circ}$ \\
Tx Power (avg.) & $96 \mathrm{~W}$ & System Loss & $1 \mathrm{~dB}$ \\
Duty Cycle & $4 \%$ & Rx Noise Temp. & $420 \mathrm{~K}$ \\
Bandwidth & $85 \mathrm{MHz}$ & PRF & $2365 \mathrm{~Hz}$ \\
Polarization & Quad (linear) & Az. Resolution & $10 \mathrm{~m}$ \\
\hline
\end{tabular}



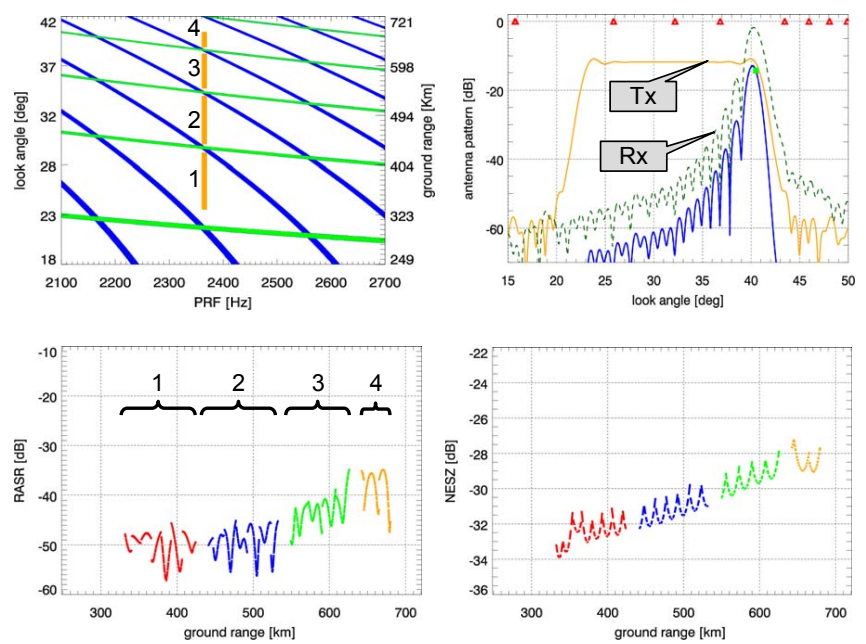

Figure 8: Performance prediction for a $350 \mathrm{~km}$ quad-pol stripmap acquisition with 4 beams. Shown are the timing diagram (upper left), the transmit and receive antenna patterns (upper right), the range ambiguities (lower left), and the NESZ (lower right) for the instrument parameters of TABLE II.

The blind ranges can be filled in by using different PRFs for each satellite pass. This shifts the data gaps to different range positions. Another opportunity to avoid blind ranges is a systematic variation of the PRF during the data take [7]. Two PRFs are sufficient to provide full wide-swath coverage in a multi-beam ScanSAR mode with only two bursts while a continuous sawtooth-like PRI variation generates for each range position even longer bursts that are interrupted by short inter-burst gaps as illustrated in Figure 9. The long bursts improve the azimuth resolution and/or provide more independent looks for interferometric applications. Very short Tx pulses may even allow for an interpolation in azimuth at the cost of a slightly increased integrated sidelobe ratio (ISLR). This could provide full stripmap resolution for a 350 km wide swath without any gaps [7].

\section{Hybrid SAR Modes}

Often, the user requirements ask for high-resolution data takes within a small area like in the immediate neighborhood of volcanoes, faults, cities or steep slopes, while at the same time continuous wide-swath coverage with a coarser resolution is needed to remove, e.g., large-scale disturbances from atmospheric signal delays. The brute force approach to meet these requirements is a continuous acquisition of very wide swaths with high geometric resolution as outlined in the previous section. However, such a global-scale high-resolution wide-swath imaging is also associated with a huge data volume, thereby increasing the demands for internal data storage, downlink, ground processing and archiving. A promising solution to these challenges is the use of new hybrid SAR modes [9]. These modes can be tailored to provide a variable resolution within the imaged scene and are hence well suited to resolve user and application conflicts without an explosion of the data volume, thereby maximizing the information about the Earth system dynamics under the constraint of a limited downlink and storage capacity.



Figure 9: Multiple swath imaging with variable PRF. Nadir echoes are sufficiently suppressed by combining the $T x$ antenna pattern with the narrow receiver beams obtained from the real-time digital beamforming.

Figure 10 shows as an example the feed activation pattern ${ }^{3}$ for one possible implementation of a hybrid SAR mode. In this example, a subset of the feed elements transmits a linear frequency modulated chirp signal of full bandwidth while the remaining feed elements transmit only a portion of the chirp with the same chirp rate. The shortened pulse durations for most of the feed elements reduce the average transmit power if compared to a full high-resolution wide-swath SAR system.
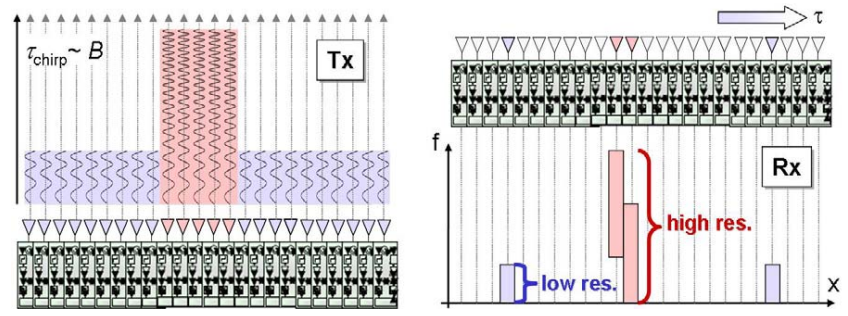

Figure 10: Feed activation patterns for a hybrid SAR mode with variable range resolution. (Left) Transmission with space-variant chirp bandwidth and pulse duration. (Right) Snapshot of the received signals in a space-frequency representation. The filled bars denote those areas where the short term power spectral density exceeds a given threshold.

The recording of the scattered signals is shown in Figure 10 on the right. Only a small subset of the feed elements receives swath echoes at a given instant of time. From these feed elements, again only a small subset receives a full bandwidth signal while the residual activated feed elements receive a short narrow-band signal that can be sampled at a much lower frequency, thereby significantly reducing the overall data volume.

The hybrid mapping of wide swaths requires in general also a systematic variation of the PRF to avoid blind ranges ${ }^{4}$. One opportunity is the combination of a local high-resolution stripmap acquisition with a wide-swath ScanSAR mode. Such a StripScan hybrid provides a non-homogeneous resolution in azimuth and allows for a further reduction of the data volume. For this, the feed elements illuminating the wide swath are operated in bursts. This reduces both the average Tx power

\footnotetext{
${ }^{3}$ Hybrid modes can also be implemented with a direct radiating array [9].

${ }^{4}$ Some applications may tolerate such gaps. A possible example is the removal of the atmospheric phase screen for large-scale deformation measurements. Low resolution SAR data acquisitions are desired during each satellite pass, but small gaps would not significantly impair the deformation and/or phase screen estimates due to their wide-ranging spatial correlations.
} 


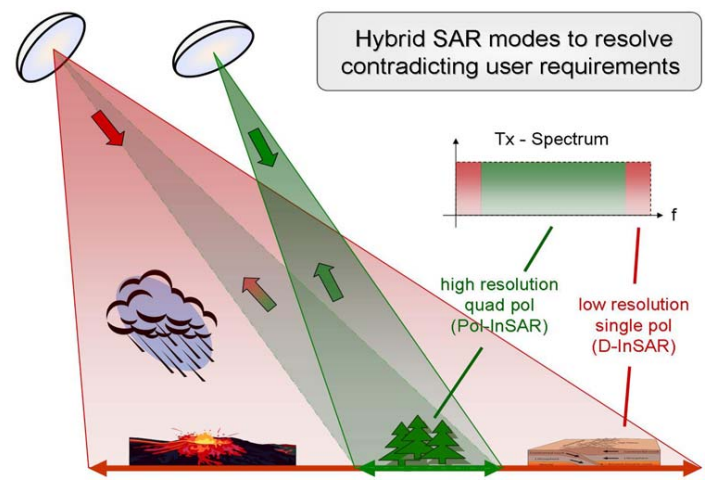

Figure 11: Hybrid SAR mode for simultaneous 3-D structure and wide swath deformation measurements.

and the number of simultaneously activated feeds during signal reception. The hybrid of stripmap and ScanSAR makes the PRF selection more stringent, since one has to avoid gaps in both the stripmap and the ScanSAR sub-swaths. Suitable PRFs can be found since the high resolution area covers only a portion of a full stripmap swath. The timing benefits moreover from the short Tx pulses and the active nadir echo suppression via the narrow Rx beams. As an alternative, one may again consider a continuous variation of the PRI as outlined in the previous section (cf. Figure 9).

An even more advanced hybrid SAR mode uses both Tandem- $\mathrm{L}$ satellites for a simultaneous scene illumination in different modes. Such a multistatic hybrid is well suited to resolve acquisition conflicts in areas that shall continuously be observed by both the deformation and the 3-D structure modes. One possible implementation divides the frequency spectrum among the different applications. As an example, one could use the central $60 \mathrm{MHz}$ of the available $85 \mathrm{MHz} \mathrm{L}-$ band spectrum for 3-D structure measurements while leaving the remaining $25 \mathrm{MHz}$ for wide swath deformation measurements as illustrated in Figure 11. The satellite within the narrow repeat-pass orbital tube illuminates the wide swath using the $25 \mathrm{MHz}$ signal in a single polarization to acquire deformation data (e.g. at half PRF using one of the previously outlined imaging modes or by using full PRF and toggling between beams illuminating different swaths) while the other satellite with the variable baseline transmits in synchrony in alternating orthogonal polarizations. The second satellite records the $60 \mathrm{MHz}$ signals from the Pol-InSAR swath while the first records in addition the $25 \mathrm{MHz}$ signals from all feed elements. The simultaneous transmission with both satellites can moreover be used to synchronize both radar systems [3].

\section{CONCLUSIONS}

This paper introduced the Tandem-L mission concept and showed its great capabilities to monitor the Earth system and its intricate dynamics. Tandem-L builds up on the know-how and experience from TanDEM-X, especially in the areas of close formation flying, system synchronization and systematic data acquisition planning. Innovative SAR imaging techniques have been developed to support a systematic data acquisition with high spatial resolution and short repeat intervals.
The advanced capabilities of Tandem-L provide also a unique opportunity to demonstrate new applications. One example is the quasi-tomographic mapping of internal 3-D structure changes of semitransparent volume scatterers via the repeated acquisition of single-pass SAR interferograms [3]. This could e.g. provide important information about structural processes in vegetation, ice, permafrost soils, etc. By this, the motto of the IEEE Radar conference 2009 "from science to systems" can also be read in reverse order and we expect a bunch of novel applications emerging from the advanced measurement capabilities. Tandem-L can hence be regarded as a first step towards a global monitoring system for the continuous observation of a highly dynamic and rapidly changing world. Radar is the optimum sensor for continuous Earth system monitoring since it provides high resolution images independent of the weather conditions at day and night. Together with its unique ability to measure subtle changes with millimetric accuracy and its even more unique ability to obtain quasi-tomographic images from space, radar will likely become the most important sensor for a huge amount of remote sensing applications, most of which we are currently even not thinking about. It is our all task to develop the best tools and techniques to be able to deal with the upcoming challenges in a rapidly changing world and environment. Tandem- $\mathrm{L}$ is a first step into this direction.

\section{ACKNOWLEDGMENT}

We thank the members of the Tandem-L science team for their valuable inputs. Many of the investigations in this paper have been performed in the framework of a close and very fruitful collaboration between DLR and NASA/JPL.

\section{REFERENCES}

[1] A. Moreira, I. Hajnsek, G. Krieger, K. Papathanassiou, M. Eineder, F. De Zan, M. Younis, M. Werner, "Tandem-L: Monitoring the Earth's Dynamics with InSAR and Pol-InSAR,” PolinSAR 2009, Frascati, Italy, January 2009.

[2] A. Freeman, et al, "Deformation, Ecosystem Structure, and Dynamics of Ice (DESDynI)," EUSAR 2008, Friedrichshafen, Germany, May 2008.

[3] G. Krieger, A. Moreira, H. Fiedler, I. Hajnsek, M. Werner, M. Younis, M. Zink, "TanDEM-X: A Satellite Formation for High Resolution SAR Interferometry," IEEE Transactions on Geoscience and Remote Sensing, vol. 45, no. 11, pp. 3317-3341, 2007.

[4] K. P. Papathanassiou and S. R. Cloude, "Single-baseline polarimetric SAR interferometry," IEEE Transactions on Geoscience and Remote Sensing, vol. 39, no. 11, pp. 2352-2363, 2001.

[5] I. Hajnsek, F. Kugler, S. Lee, K. Papathanassiou, "Tropical-Forest-Parameter Estimation by Means of Pol-InSAR: The INDREX-II Campaign," IEEE Trans. Geoscience and Remote Sensing, vol. 47, no. 2, pp. 481-493, 2009.

[6] A. Monti Guarnieri and S. Tebaldini, "Hybrid Cramér-Rao Bounds for Crustal Displacement Field Estimators in SAR Interferometry," IEEE Signal Processing Letters, vol. 14, no. 12, December 2007.

[7] G. Krieger, N. Gebert, M. Younis, A. Moreira, "Advanced Synthetic Aperture Radar Based on Digital Beamforming and Waveform Diversity," IEEE Radar Conference, pp. 767-772, Rome, Italy, May 2008.

[8] A. Freeman, G. Krieger, P. Rosen, M.Younis, W.T.K. Johnson, S. Huber, R. Jordan, A. Moreira, "SweepSAR: Beam-forming on Receive using a Reflector-Phased Array Feed Combination for Spaceborne SAR," IEEE Radar Conference, Pasadena, USA, May 2009.

[9] G. Krieger, N. Gebert, A. Moreira, "Multidimensional Waveform Encoding: A New Digital Beamforming Technique for Synthetic Aperture Radar Remote Sensing," IEEE Transactions on Geoscience and Remote Sensing, vol. 46, no. 1, pp. 31-46, January 2008. 